\title{
Influencia de la biblioterapia sobre el perfeccionismo infantil
}

\author{
The influence of bibliotherapy on child perfectionism
}

Leonardo Andrés Aguilar Durán*

Isaac Daniel Depablo Marrero**

\section{Resumen}

El perfeccionismo es un rasgo de personalidad caracterizado por la fijación de estándares de rendimiento excesivamente altos. Varios estudios han demostrado la relación entre perfeccionismo y malestar psicológico en niños y adolescentes. El objetivo de este trabajo fue determinar la efectividad de la biblioterapia basada en literatura infantil -más propiamente, literapia- para disminuir los niveles de perfeccionismo en los niños. Se realizó una investigación de campo con alcance explicativo y diseño cuasiexperimental pre-post de un solo grupo. Se aplicó un taller biblioterapéutico breve a 16 niños con puntajes extremos en perfeccionismo $\left(\geq \mathrm{P}_{75}\right)$, quienes cursaban $4 .^{\circ}$ y $5 .^{\circ}$ grado de educación primaria -i.e., niños de 9 a 11 años de edad- en un colegio privado de Caracas. La selección literaria empleada constó de ocho cuentos infantiles escritos e ilustrados por autores europeos y americanos. El perfeccionismo se evaluó con la versión venezolana (Aguilar \& Castellanos, 2017) de la Escala de Perfeccionismo Infantil (Oros, 2003). La biblioterapia logró disminuciones estadísticamente significativas en las puntuaciones de los niños en perfeccionismo y sus dimensiones $(p<.01)$. Estos resultados coincidieron con la evidencia empírica previa referida por investigaciones foráneas. Se recomienda la aplicación de la terapia de lectura en contextos similares. Podrían realizarse ciertos ajustes para potenciar sus efectos y mejorar el control experimental.

Palabras clave: perfeccionismo infantil, biblioterapia, literapia, literatura infantil, niños venezolanos.

Abstract
Perfectionism is a personality disposition
characterized by the setting of excessively
high performance standards. Several
studies have proved the relationship
between perfectionism and psychological
maladjustment in children and adolescents.

\section{Abstract}

*Universidad Pedagógica Experimental Libertador (UPEL), Instituto Pedagógico de Caracas (IPC), Venezuela. Mail de contacto: psileonardo@gmail.com

**Universidad Pedagógica Experimental Libertador (UPEL), Instituto Pedagógico de Caracas (IPC), Venezuela. Mail de contacto: isaacdepablo@gmail.com DOI: https://doi.org/10.46553/RPSI.16.32.2020.p24-45

Fecha de recepción: 16 de noviembre de 2019 - Fecha de aceptación: 18 de mayo de 2020 
The aim of this study was to determine the effectiveness of bibliotherapy based on children's literature -more properly, literature therapy- on perfectionism levels in children. A quantitative investigation with explanatory scope and quasi-experimental design (pretest-posttest, one group) was carried out. A brief program of bibliotherapy was applied to 16 children with extreme perfectionism $(\geq$ $\mathrm{P}_{75}$ ), who were in $4^{\text {th }}$ and $5^{\text {th }}$ grade of primary education-i.e., children aged 9-10-in a private school in Caracas. The literary selection used consisted of eight children's stories written and illustrated by European and American authors. Perfectionism was evaluated with the Venezuelan version (Aguilar \& Castellanos, 2017) of the Scale of Childhood Perfectionism (Oros, 2003). Bibliotherapy achieved statistically significant decreases in the scores of children in perfectionism and their dimensions $(p<.01)$. These results coincide with previous empirical evidence referred by foreign investigations. The application of reading therapy in similar contexts is recommended. Certain adjustments could be made to maximize its effects and improve experimental control.

Key words: child perfectionism, bibliotherapy, literature therapy, children's literature, Venezuelan children.

\section{Introducción}

El perfeccionismo es una disposición de la personalidad que se caracteriza por el establecimiento de niveles de exigencia muy elevados, baja tolerancia a los errores y la tendencia a evaluar críticamente el desempeño (Flett \& Hewitt, 2002).

El interés por estudiar el perfeccionismo en los niños ha ido en aumento. De hecho, se sabe que ya durante la infancia se asocia con condiciones psicopatológicas tales como: depresión, ansiedad, trastorno obsesivo-compulsivo y trastornos de la conducta alimentaria (Douilliez \& Hénot, 2013; Dry, Kane, \& Rooney, 2015; Elizathe, Murawski, Custodio, \& Rutsztein, 2012; Elizathe et al., 2011; Huggins, Davis, Rooney, \& Kane, 2008; Mitchell, Newall, Broeren, \& Hudson, 2013; Nobel, Manassis, \& WilanskyTraynor, 2012; Soreni et al., 2014; Stornelli, Flett, \& Hewitt, 2009; Wolters et al., 2011).

También en la edad infantil, altos niveles de perfeccionismo se han relacionado con otros problemas de salud mental como es el caso de la conducta agresiva en sus componentes cognitivo -hostilidad-, fisiológico-emocional -ira- y motor (GarcíaFernández, Vicent, Inglés, Gonzálvez, \& Sanmartín, 2017; Hewitt et al., 2002; Vicent, Inglés et al., 2019b; Vicent, Inglés, Sanmartín, Gonzálvez, \& García-Fernández, 2018). Además, los niños perfeccionistas pueden estar más expuestos al malestar psicológico debido a la carencia de algunos recursos cognitivos que les servirían para desarrollar un afrontamiento efectivo del estrés (Oros, 2005).

Uno de los ámbitos donde el perfeccionismo tiene implicaciones importantes es el educativo, especialmente debido a su asociación con variables motivacionales, así como con cogniciones y conductas vinculadas al logro (Bong, Hwang, Noh, \& Kim, 2014).

En tal sentido, los resultados del estudio de Vicent, Inglés et al. (2019a) evidenciaron la tendencia de los niños con elevados niveles de perfeccionismo a atribuir sus fracasos a la falta de esfuerzo propio. 
Basados en conclusiones derivadas de investigaciones con población adulta (Egan, Piek, Dyck, \& Rees, 2007; Hewitt, Flett, \& Mikail, 2017; Stoeber, Schneider, Hussain, $\&$ Matthews, 2014), los autores ven probable que el patrón atribucional antedicho pueda desencadenar una serie de consecuencias emocionales aversivas. En concreto indican que, cuando los niños con un perfeccionismo extremo afrontan el fracaso, responden con diversas formas de autocastigo: se sienten culpables, se critican con severidad y experimentan emociones de tono negativo como remordimiento, vergüenza y tristeza. Además, en estos niños la sensación de fracaso se presenta con mayor frecuencia, ya que tienen una visión sesgada de los aciertos y las equivocaciones que les lleva a interpretar su desempeño en términos de "todo o nada". Por lo tanto, el alumnado con perfeccionismo alto podría ser más vulnerable psicológicamente tras sucesivos fracasos. De allí que se recomiende a psicólogos y docentes que administren intervenciones escolares dirigidas a que los estudiantes perfeccionistas interpreten sus éxitos y fallas de manera realista, contrarresten la autocrítica mediante el desarrollo de la resiliencia y la autocompasión, y se mantengan optimistas con respecto a su rendimiento futuro (Flett \& Hewitt, 2014; Vicent, Inglés et al., 2019a).

En el contexto de la educación primaria, el perfeccionismo es también una variable que influye en la ansiedad escolar (Inglés, García-Fernández, Vicent, Gonzálvez, \& Sanmartín, 2016; Vicent, Gonzálvez et al., 2019) y en el rechazo a la escuela (Gonzálvez, Sanmartín, GarcíaFernández, Inglés, \& Vicent, 2016), igualmente es considerado un factor de riesgo para ser víctima de acoso escolar
(Morán, García, \& Fínez, 2015). Se remite al lector interesado a consultar la revisión que ofrecen Chemisquy, Oros, Serppe y Ernst (2019) del perfil que muestran los niños con un perfeccionismo exacerbado, no solo en relación con situaciones académicas, sino en el área de las relaciones sociales y en referencia al ámbito lúdico-deportivo.

Ahora bien, aun cuando existe evidencia convincente sobre lo pernicioso que resulta el perfeccionismo en los niños (véase, para una revisión, Flett \& Hewitt, 2012), diversos autores (e.g., Gonzálvez, Inglés, Lagos-San Martín, GarcíaFernández, \& Martínez-Monteagudo, 2015; Morris \& Lomax, 2014) coinciden en apreciar que, frente al interés que ha generado estudiar este atributo en adultos, el perfeccionismo infantil constituye un área de investigación todavía incipiente y que solo un número relativamente pequeño de estudios se ha centrado en desarrollar y comprobar formas de tratamiento útiles para abordarlo, escasez que es sobre todo patente en el contexto latinoamericano. En parte, esto se debe a que el perfeccionismo puede pasar desapercibido en los niños, y no solo porque algunos intenten ocultar el malestar que experimentan ante el fracaso (Flett \& Hewitt, 2013), sino debido a que el esfuerzo por la excelencia a menudo es aupado en el entorno escolar.

En Venezuela, Aguilar y Castellanos (2016a, 2016b, 2017) llevaron a cabo una serie de estudios psicométricos que les permitieron adaptar y validar la Escala de Perfeccionismo Infantil de Oros (2003) a la población infantil venezolana. Recientemente, haciendo uso de este instrumento, Aguilar (2019) encontró niveles moderados de perfeccionismo en los niños caraqueños, los cuales representan 
cierto riesgo al bienestar y pueden poner en peligro el buen desarrollo psicosocial de los escolares, habida cuenta de los desórdenes emocionales con los que aquel se vincula. En dicha investigación también se logró poner en evidencia que los participantes con alto estrés obtuvieron puntuaciones superiores en perfeccionismo y aquellos con alto perfeccionismo presentaron mayores puntuaciones en estrés. Se trata de hallazgos que constituyen un primer acercamiento a la relación entre perfeccionismo y malestar psicológico en el caso de los niños venezolanos, a partir de los cuales se destaca la necesidad de diseñar estrategias orientadas a abordar esta problemática y a prevenir sus consecuencias negativas durante la infancia, etapa en la cual determinados comportamientos parentales -e.g., las exigencias de perfección- promueven su desarrollo (Chemisquy et al., 2019).

Una de las prioridades actuales de la investigación en ciencia psicológica es la elaboración de estrategias de prevención e intervención basadas en la evidencia (Chemisquy, 2018). Como se adelantaba, la realidad no es distinta en materia de perfeccionismo. De acuerdo con el balance realizado por Vicent (2017), pese a que este atributo constituye una condición de riesgo para la salud mental y a pesar de que ejerce un impacto negativo en el tratamiento de diversas psicopatologías, no son muy abundantes los estudios que evalúan la eficacia de una intervención para el perfeccionismo con ciertas garantías, menos aun en niños y adolescentes, en quienes un abordaje del problema podría ser más efectivo, ya que, como indica Nobel (2007), los jóvenes todavía están desarrollando su personalidad y su perfeccionismo puede no ser tan estable como en los adultos. Vicent (2017) describe los tratamientos que se han desarrollado para prevenirlo y superarlo: el tratamiento psicodinámico, el tratamiento cognitivoconductual, la realimentación, la terapia de juego, el mindfulness, los programas de intervención escolar e, incluso, la medicación. Según la autora, de las opciones propuestas, la terapia cognitivo-conductual es la más empleada para la prevención y la reducción del perfeccionismo.

Otra de las técnicas utilizadas para hacer frente al perfeccionismo y sobre la cual ha habido un creciente interés en los últimos años es la biblioterapia, misma que - en sentido amplio - se refiere a la lectura de todo material bibliográfico con fines terapéuticos (Botelho, 2013). Bueno y Caldin (2002) la definen como "un proceso dinámico de interacción entre el lector, el texto y el oyente, que ayuda en el crecimiento emocional y psicológico" (p. 158). Cuando el insumo de este proceso terapéutico lo constituyen textos de naturaleza literaria, incluyendo la poesía, se denomina con mayor propiedad literapia (Silverberg, 2003). Se trata de un método que, si bien hunde sus raíces en la teoría psicodinámica (Rincón, 2013), actualmente tiene aplicaciones basadas no solo en principios psicoanalíticos (e.g., Marín, 2009), sino también en técnicas racionales de la ingeniería conductual (Montgomery, 2002), e incluso en el mindfulness (e.g., Wimberley, Mintz, \& Suh, 2016) y la logoterapia (Arias, Miguez, Molfino, \& Rega, 2000).

En la actualidad, existen debates con respecto a los usos de la biblioterapia y a la manera en que se debería impartir. En este sentido, los estudiosos de la materia han recurrido a diferentes taxonomías para 
clasificar los tipos de biblioterapias. En algunos casos, la nomenclatura obedece al contexto donde la intervención se realiza -e.g., centros de salud, ámbito educativo- y, en otros casos, depende de los materiales que se utilizan -e.g., libros de autoayuda y desarrollo personal, obras literarias de varios géneros-. Lo cierto es que, como bien señalan Castro y Altamirano (2016), la biblioterapia -en sus distintas vertientes- se ha afianzado en décadas recientes como un atractivo modelo educativo-terapéutico. Constituye una estrategia educativa debido a que la lectura de cualquier tipo proporciona nueva información e instruye; y resulta terapéutica, dado que, al mejorar el estado anímico, incide en el bienestar de quienes participan en los talleres de lectura -incluyendo participantes considerados sanos, pero que, como el común de las personas, están expuestos a numerosos estresores en diversas áreas de la vida-.

Babarro y Lacalle (2018) realizaron una investigación de carácter documental con el fin de analizar los efectos beneficiosos de la biblioterapia en el proceso de saludenfermedad del niño en diferentes momentos y contextos. La primera categoría que destacan es la referida a los niños sanos, es decir, aquellos sin una enfermedad manifiesta y reconocible, en quienes la terapia de lectura puede ayudar a hacer frente a las adversidades propias del desarrollo infantil. El segundo eje de acción de la biblioterapia se enmarca en las alteraciones mentales que con frecuencia aparecen durante la infancia. La tercera categoría que emergió en el mencionado estudio se refiere a la estancia prolongada en un hospital. Frente al advenimiento de esta situación $\tan$ estresante, se dice que la literatura favorece el bienestar emocional en los niños, permitiéndoles verse a sí mismos como seres completos y no solo como enfermos. La cuarta posibilidad de aplicación de la biblioterapia es ante patologías físicas que pueden afectar a los niños en determinadas ocasiones. En estos casos, se ayuda al niño a minimizar la afectación emocional con que suelen cursar tales enfermedades. En efecto, a través de historias cargadas de humor, aventura y suspenso, los lectores pueden tener acceso a realidades alternativas que les permiten distraerse de sus preocupaciones, sentirse acompañados y tender puentes de comunicación con sus pares y familiares. Por último, se reporta literatura con la que se ofrece seguridad emocional y acompañamiento en el proceso de muerte del niño.

En la mayoría de los países de América Latina, sin embargo, el uso de biblioterapia ha sido limitado (para una revisión, véase Camero, 2016). Desde una perspectiva bibliotecológica, Gómez (2011) concluyó que la práctica biblioterapéutica no había sido tomada en cuenta como un posible servicio en Venezuela. La autora también resaltó que el campo de la biblioterapia no se había contemplado en los planes de estudio de Bibliotecología a nivel nacional, ni tampoco se conocía de alguna oferta formativa sobre esta disciplina en el país. Entre los estudios que, en el caso venezolano, aparecen citados con mayor frecuencia se cuentan: el programa biblioterapéutico para niños víctimas de maltrato diseñado por Marín (2009) desde el enfoque psicodinámico y la experiencia reportada por Pérez de Rosell (2011) sobre literapia con niños hospitalizados. A este panorama debe agregarse que, tanto Babarro y Lacalle 
(2018), como Castro y Altamirano (2016), destacan que la mayoría de los estudios sobre los efectos de la biblioterapia - en especial aquellos provenientes de Latinoaméricason descriptivos y cualitativos, motivo por el cual deberían realizarse más estudios experimentales o cuasiexperimentales.

La biblioterapia podría ser una técnica particularmente útil para los niños perfeccionistas porque estos suelen ser chicos con capacidades académicas sobresalientes, razón por la cual es posible que adquieran tempranamente habilidades de lectura -e.g., rapidez integrativa, concentración extendida, persistencia ante la dificultad para la comprensión- y otras habilidades cognitivas -e.g., curiosidad; pensamiento abstracto; procesos cognitivos de alto nivel como análisis, síntesis y evaluación-, a lo que se suma la posibilidad de que sean lectores entusiastas que obtengan un sincero placer de la lectura (İlter, Leana-Taşcılar, \& Saltukoğlu, 2018). Además, se considera que la biblioterapia les proporcionaría un entorno seguro para entender sus pensamientos y sentimientos, en el cual explorar, discutir y evaluar sus miedos. Por ejemplo, las discusiones grupales durante la biblioterapia podrían ayudar a los niños a comprender que no están solos, a que se den cuenta de que otros compañeros tienen experiencias emocionales similares a las suyas.

A pesar de las consideraciones destacadas, escasas investigaciones (Ilter et al., 2018; Mofield \& Chakraborti-Ghosh, 2010; Zousel, Rule, \& Logan, 2013) han empleado la literapia con niños que ansían la perfección. Las que lo han hecho son todas investigaciones foráneas -concretamente de Estados Unidos y Turquía-, dos de ellas trabajaron con niños superdotados $\mathrm{y}$, en uno de los casos, la biblioterapia fue una estrategia que formó parte de un programa curricular de mayor alcance. Por otra parte, si bien existen numerosos libros de autoayuda para combatir el perfeccionismo (e.g., Shafran, Egan, \& Wade, 2010; Somov, 2010), claramente los mismos están dirigidos a un público adulto y cuentan con pocos estudios empíricos que avalen la eficacia que prometen (e.g., Wimberley et al., 2016). Fuentes consultadas también reflejan la utilización de la biblioterapia de autoayuda e informativa -con fines de psicoeducación- como complemento en el tratamiento de diversos trastornos asociados al perfeccionismo, lo que pone de relieve la subutilización de esta técnica terapéutica y su raro uso como estrategia principal o de cabecera dentro de un proceso clínico. Precisamente por esta razón, la mejoría de los individuos en el caso precitado no podría atribuirse por entero al procedimiento biblioterapéutico.

A modo de síntesis, puede decirse que la naturaleza desadaptativa del perfeccionismo infantil y los peligros que este conlleva justifican la puesta en marcha de estrategias de prevención y de tratamiento para niños en riesgo. En este sentido, a pesar de su poca aplicación en el contexto local, la biblioterapia se presenta como una técnica de intervención especialmente pertinente para la población meta y con necesidad de verificación empírica, pues sobre ella predominan informes de tipo anecdótico. Este estudio se realizó con la finalidad de determinar si la administración de un taller biblioterapéutico -o más bien literapéutico, por estar basado en la lectura de textos literarios- tenía una influencia significativa en la disminución de los 
niveles de perfeccionismo en niños de proceso educativo. educación primaria. En razón de ello, y hasta donde se tiene conocimiento, constituye el primer estudio de su tipo en Latinoamérica. De manera tangencial, este planteamiento resalta el importante papel que podría jugar la escuela en la detección y prevención del perfeccionismo como factor de vulnerabilidad psicológica durante la infancia, al mismo tiempo que reafirma la relevancia que variables socioemocionales -como el perfeccionismo- tienen en el

\section{Tipo y diseño de investigación}

Fue una investigación de campo, con enfoque cuantitativo y alcance explicativo (Hernández, Fernández, \& Baptista, 2014). Se utilizó un diseño cuasiexperimental de pre y postest con un solo grupo (Montero \& León, 2007).

Tabla 1

Participantes clasificados según sexo, edad y escolaridad

\begin{tabular}{ccccccc}
\hline \multirow{2}{*}{ Edad } & \multicolumn{2}{c}{$4 .^{\circ}$ grado } & & \multicolumn{2}{c}{$5 .^{\circ}$ grado } & \\
\cline { 2 - 3 } \cline { 5 - 6 } & Chicos & Chicas & & Chicos & Chicas & Totales \\
\hline 9 años & 3 & 1 & & - & - & $4(25 \%)$ \\
10 años & 3 & - & & 3 & 2 & $8(50 \%)$ \\
11 años & - & - & & 2 & 2 & $4(25 \%)$ \\
Totales & $6(37.5 \%)$ & $1(6.2 \%)$ & & $5(31.2 \%)$ & $4(25.0 \%)$ & $16(100 \%)$ \\
\hline
\end{tabular}

\section{Participantes}

El grupo de participantes estuvo constituido por 16 niños, de ellos 11 eran chicos $(68.7 \%)$ y 5 eran chicas $(31.2 \%)$. La edad promedio fue de 10 años $(D E=0.73)$. Estaban cursando $4 .^{\circ}(n=7,43.7 \%)$ y $5 .^{\circ}$ $(n=9,56.2 \%)$ grado de primaria, durante el año escolar 2019-2020, en un colegio privado de Caracas, Venezuela. En la tabla 1 puede apreciarse la distribución del grupo en función del sexo, la edad y el grado de estudio.

\section{Instrumento}

Se empleó la versión adaptada y validada por Aguilar y Castellanos (2016a, 2016b, 2017) al contexto venezolano de la Escala de Perfeccionismo Infantil de Oros (2003), de origen argentino. Está constituida por 15 ítems que evalúan cuatro dimensiones del constructo: a) Autodemandas Absolutistas (AA), b) Autodemandas Específicas (AE), c) Malestar ante el Fracaso (MF) y d) Correlatos Negativos de los Errores (CNE). El instrumento se contesta en una escala tricotómica tipo Likert: $1=$ no/no lo pienso, 2 $=a$ veces/lo pienso algunas veces, y $3=$ síllo pienso muchas veces. Un Análisis Factorial Exploratorio con rotación ortogonal varimax mostró que los cuatro factores explican $50.27 \%$ de la varianza total. Por su parte, en un estudio de validez convergente, el perfeccionismo se asoció con el estrés ( $r$ $=.36, p<.01)$, mientras que los correlatos 
negativos de las equivocaciones mostraron una correlación baja pero significativa con el temor a hablar en público $(r=.18, p<$ $.05)$. Los valores del alfa de Cronbach para la escala total y la dimensión AA resultaron satisfactorios $(\alpha=.80)$; las dimensiones $\mathrm{AE}$ $(\alpha=.61)$, MF $(\alpha=.67)$ y $\mathrm{CNE}(\alpha=.64)$ presentaron índices de consistencia interna que Prieto y Muñiz (2000) califican de adecuados para fines de investigación pero con algunas carencias, quizás debido al bajo número de ítems de estas facetas.

\section{Procedimiento}

En primer lugar, se realizó una reunión con la directora del centro educativo, a quien se expuso el objetivo de este trabajo. Aprobada la propuesta, a través de una carta informativa se pidió a los padres de los niños la firma de un consentimiento informado. La decisión de escoger escolares de $4 .^{\circ}$ y $5 .^{\circ}$ grado se tomó considerando la pertinencia etaria de la literatura seleccionada, así como también la madurez cognitiva y las habilidades de comprensión lectora necesarias, tanto para entrar en contacto con la selección de literatura realizada, como para seguir las instrucciones que implicaban la participación en el taller diseñado y la contestación del instrumento de medición. Este último aspecto había sido valorado en experiencias de trabajo previas.

Los niños completaron las pruebas voluntariamente en sesiones grupales de aproximadamente $15 \mathrm{~min}$, mismas que tuvieron lugar en los salones de clase y en horario escolar, contando con la autorización de los docentes encargados. Previamente, el procedimiento a seguir para rellenar los cuestionarios fue informado por los investigadores, quienes en todo momento trataron a los participantes con apego a los estándares éticos que la investigación científica exige (Santalla \& González, 2011). 16 niños conformaron el grupo experimental y participaron en el taller biblioterapéutico organizado. Se trató de los estudiantes que mostraron un mayor nivel de perfeccionismo en el pretest (con puntuaciones $\geq$ al centil 75) y que, al mismo tiempo, expresaron agrado por la lectura, tópico que se había explorado en el cuestionario mediante la pregunta "¿Qué tanto te gusta leer cuentos?" y las opciones nada, un poco y mucho. De esta manera, se eligieron los chicos que con más urgencia necesitaban ser intervenidos terapéuticamente y los que, a su vez, podrían obtener un mayor beneficio del abordaje planteado. La $n$ fue la que se posibilitó con los recursos materiales y humanos disponibles.

Una vez formado el grupo de intervención, se procedió a administrar el taller cuya efectividad se investigaba, lo cual ocurrió en un espacio con las comodidades requeridas dispuesto por la institución educativa. La selección literaria empleada constó de ocho cuentos infantiles escritos e ilustrados por autores provenientes de Estados Unidos, Reino Unido, Francia, España y Argentina. Un par de libros habían sido utilizados con propósitos similares al actual en los estudios de İlter et al. (2018) y de Zousel et al. (2013); los demás títulos fueron sugeridos por lectores adultos de literatura infantil, quienes atendieron a la solicitud de recomendaciones difundida por los investigadores en varios grupos de Facebook, a los cuales se adscribían aficionados a ese tipo de literatura.

El taller de biblioterapia constó de tres sesiones, cada una con una duración 
de aprox. $2 \mathrm{~h}$ y media. La primera sesión tuvo como objetivos a) que los participantes comprendieran que el deseo de hacer las cosas de manera perfecta supone un freno a la creatividad y b) que advirtieran las ventajas de tolerar un cierto grado de incertidumbre y ambigüedad en sus vidas -tras percatarse del malestar que trae aparejado la necesidad de control sobre el entorno-. Con tales propósitos se leyeron los libros El punto (Reynolds, 2003), Alexander y el día terrible, horrible, espantoso, horroroso (Viorst \& Cruz, 1972/1991) y Así es la vida (Ramírez \& Ramírez, 2005). La segunda sesión persiguió c) que los niños apreciaran las experiencias emocionales positivas que pueden surgir cuando se abandonan los intentos por hacer las cosas de forma perfecta y d) que entendieran que un "desempeño perfecto" - especialmente de acuerdo con reglas externas - no es el único criterio de éxito. Estos objetivos fueron abordados con los libros Casi (Reynolds, 2004), Sofía, la vaca que amaba la música (De Pennart, 1999/2011) y Las jirafas no pueden bailar (Andreae \& Parker-Rees, 1999/2009). Por último, en la tercera sesión se intentó e) que los participantes adoptaran una perspectiva más flexible en la esfera de la moralidad y f) que valoraran el hecho de cometer errores como parte esencial del proceso de aprendizaje, pese a recibir demandas externas de "conductas perfectas". El alcance de dichas metas se posibilitó a través de los libros Petit, el monstruo (Misenta, 2010) y La niña que nunca cometía errores (Pett \& Rubinstein, 2013).

Cada uno de los encuentros comenzaba con el facilitador de turno presentando -o recordando- las reglas del aula, después de lo cual leía el libro que correspondía desde un monitor digital, mientras los participantes seguían el texto en pantalla. Posteriormente, el tallerista hacía preguntas sobre la historia y se producía una discusión sobre los aspectos del perfeccionismo relacionados con el relato.

A modo de ejemplo, los tópicos de la sobreorganización y el miedo a cometer errores fueron abordados con el cuento $L a$ niña que nunca cometía errores mediante preguntas disparadoras como las siguientes: ¿cómo era Beatriz antes de cometer su error?, ¿qué ventajas tiene ser tan ordenados como Beatriz? o ¿qué ventajas tiene el orden para nuestras vidas?, ¿y qué desventajas tiene el hecho de ser tan organizados?, ¿cómo se sentía Beatriz después de cometer su "casi-error"?, ¿cómo se sentía mientras se preparaba para el espectáculo de talentos?, ¿cómo se sentía momentos antes de su actuación?, ¿cómo se sintió la niña justo al equivocarse en pleno escenario?, ¿se han sentido así?, ¿por qué será que la noche de su equivocación "Beatriz durmió mejor que nunca"?, ¿cómo cambió la vida de Beatriz luego de su primer error?, ¿por qué creen que ocurrió esto?, ¿qué aprendió Beatriz con respecto a los errores?, etcétera. Al finalizar la discusión los estudiantes estuvieron en capacidad de identificar las consecuencias negativas de la presión a la que alguien se somete cuando quiere ser perfecto en todo $y$, particularmente, los efectos adversos que acarrea una excesiva preocupación por los errores, tarea en la que fue primordial la comparación entre las características de Beatriz antes y después de aceptar su error: de ser una chica rutinaria, muy organizada y meticulosa, angustiada y que se divertía poco, pasó a dormir mejor, estar más relajada y disfrutar más de la vida, los amigos, los 
juegos y las risas compartidas. Especial atención recibió el hecho de que Beatriz haya afrontado el error riéndose de sí misma. En suma, se propició que los niños entendieran que los errores son la base del aprendizaje.

Después de la lectura y la discusión se realizaban una serie de actividades -expresión escrita, dibujo, baile y cineforo-, las cuales se desarrollaron en medio de un clima lúdico-recreativo que se buscó gestar con el fin de aminorar en los sujetos la conciencia de estar siendo estudiados. Al culminar la intervención se realizó el postest, actividad que siguió el procedimiento estandarizado del pretest en todo cuanto le fue aplicable.

\section{Análisis de datos}

En primer lugar, se evaluó la normalidad y la homocedasticidad de los datos con las pruebas de Shapiro-Wilk (ya que $n<30$ ) y de Levene, respectivamente. En los casos en que se logró comprobar normalidad -tanto en la distribución del pretest como en la del postest- y homogeneidad de varianzas, la prueba $t$ de Student para muestras relacionadas permitió saber si se habían producido diferencias estadísticamente significativas entre las puntuaciones obtenidas por los niños antes y después del taller biblioterapéutico impartido. En cambio, cuando alguno de los mencionados criterios no se cumplió, la prueba de Wilcoxon para pares de rangos fue la que hizo posible determinar si las diferencias encontradas resultaban significativas. Como medidas del tamaño del efecto, se emplearon - según correspondíauna variante de la $d$ de Cohen para muestras apareadas (Dunlap, Cortina, Vaslow, \&
Burke, 1996) y la delta de Cliff $(\delta C)$. Todos los análisis se realizaron utilizando SPSS versión 23 , excepto el cálculo del tamaño de los efectos: la $d$ de Cohen se obtuvo con ayuda de una calculadora en línea y el delta de Cliff mediante el programa Cliff's Delta Calculator (CDC: Macbeth, Razumiejczyk, \& Ledesma, 2011).

\section{Resultados}

Como puede observarse en la tabla 2, el valor del nivel crítico que muestra el contraste estadístico inferencial ha permitido rechazar la hipótesis de igualdad de medias y concluir que el nivel de perfeccionismo después de la intervención fue significativamente menor que el nivel exhibido en el pretest $t_{(15)}=8.47$, $p<.01, d=2.44)$. Claramente, la magnitud del efecto resultó muy relevante.

De igual manera, se hizo posible rechazar la hipótesis de igualdad de promedios para las dimensiones del perfeccionismo y concluir que entre ambas medidas -pretest y postest- existían diferencias significativas: autodemandas absolutistas $(Z=-3.45, p<$ $.01, \delta C=0.87)$, autodemandas específicas $(Z$ $=-2.77, p<.01, \delta C=0.59)$, malestar ante el fracaso $(Z=-2.89, p<.01, \delta C=0.59)$ y correlatos negativos de los errores $\left(t_{(15)}=\right.$ $6.02, p<.01, d=1.47)$. Dado que las medias en los cuatro factores fueron superiores en el pretest de comparación, se considera que la aplicación del taller trajo consigo una mejoría notable para los participantes. Los tamaños de los efectos resultaron grandes en todos los casos.

\section{Discusión}

Pese a que el perfeccionismo de niños 
Tabla 2

Resultados pretest/postest en la Escala de Perfeccionismo Infantil aplicada al grupo de estudio

\begin{tabular}{|c|c|c|c|c|c|c|c|}
\hline \multirow[b]{2}{*}{ Variable } & \multicolumn{2}{|c|}{ Pretest } & \multicolumn{2}{|c|}{ Postest } & \multirow[b]{2}{*}{ Estadístico } & \multirow[b]{2}{*}{ 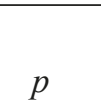 } & \multirow[b]{2}{*}{ TE } \\
\hline & $M$ & $D E$ & $M$ & $D E$ & & & \\
\hline Perfeccionismo & 35.25 & 3.59 & 26.06 & 3.94 & $t=8.469$ & $.000^{* *}$ & $d=2.44$ \\
\hline AA & 15.06 & 1.06 & 10.94 & 2.41 & $Z=-3.449$ & $.001^{* *}$ & $\delta C=0.87$ \\
\hline $\mathrm{AE}$ & 4.75 & 1.39 & 3.44 & 0.73 & $Z=-2.767$ & $.006^{* *}$ & $\delta C=0.59$ \\
\hline MF & 6.75 & 1.29 & 5.31 & 1.14 & $Z=-2.891$ & $.004^{* *}$ & $\delta C=0.59$ \\
\hline $\mathrm{CNE}$ & 8.69 & 1.78 & 6.38 & 1.20 & $t=6.018$ & $.000^{* *}$ & $d=1.47$ \\
\hline
\end{tabular}

Nota: $\mathrm{AA}=$ Autodemandas Absolutistas, $\mathrm{AE}=$ Autodemandas Específicas, $\mathrm{MF}=$ Malestar ante el Fracaso, $\mathrm{CNE}=$ Correlatos Negativos de los Errores. Pruebas de comparación: $t$ para muestras correlacionadas y $Z$ de Wilcoxon, según normalidad y homocedasticidad de las variables. TE $=$ tamaño del efecto.

$* * p<.01$.

$\mathrm{y}$ adolescentes es un tema discutido con cierta frecuencia, solo un número limitado de estudios ha abordado el problema de cómo lidiar con sus aspectos negativos. Además, aunque ha venido en aumento la cantidad de estudios sobre el efecto de los enfoques terapéuticos cognitivos sobre el perfeccionismo infantojuvenil, se han realizado apenas unas pocas investigaciones acerca de los efectos de la biblioterapia en niños perfeccionistas de Norteamérica y Turquía. El arqueo bibliográfico efectuado develó que, si bien a primera vista el perfeccionismo puede ser considerado como una característica positiva de los jóvenes estudiantes, la verdad es que tiene toda una serie de consecuencias negativas sobre la salud de los más pequeños, hecho que hace aún más urgente la necesidad de desarrollar programas de prevención y tratamiento en el medio local. Por lo tanto, el objetivo de la presente investigación fue determinar la influencia de la administración de un taller biblioterapéutico sobre los niveles de perfeccionismo en un grupo de niños escolarizados de Caracas, Venezuela.

Lo primero que se hizo fue identificar a los niños candidatos a beneficiarse de la intervención psicoeducativa propuesta para el manejo del perfeccionismo. Además del criterio clínico, primó el hecho de que el grupo elegido para este estudio estuviera compuesto por estudiantes que disfrutaban cotidianamente de la lectura. También, desde un principio, se pensó la manera en que se podría configurar el taller de biblioterapia. El repertorio de libros infantiles utilizado se recabó a través de dos vías: la búsqueda de títulos que contaran con el aval empírico necesario según el propósito perseguido y la consulta a lectores adultos aficionados a la literatura para niños. El taller fue conducido por ambos investigadores en las tres sesiones previstas, tras lo cual se evaluó su 
efectividad.

Como era de esperarse, se encontró una disminución significativa entre los resultados del pretest y el postest del grupo experimental. Esta reducción no se limitó a las puntuaciones totales de la Escala de Perfeccionismo Infantil, sino que también se observó en las subescalas correspondientes. Indirectamente, al haber obtenido evidencia empírica acerca de los efectos positivos de la biblioterapia sobre el perfeccionismo que se presenta a temprana edad, la selección de literatura infantil que se utilizó quedó validada como material de uso terapéutico.

Los hallazgos del estudio actual apoyan la presunción de Nugent (2000) acerca de que la biblioterapia tendría un papel útil que desempeñar en el caso de estudiantes perfeccionistas. También son compatibles con las conclusiones a las que llegaron los únicos estudios que se han realizado en el área (İter et al., 2018; Mofield \& Chakraborti-Ghosh, 2010; Zousel et al., 2013), los cuales crearon programas a través de los que investigaron los efectos de la biblioterapia en el perfeccionismo de niños y adolescentes.

Asímismo, los resultados expuestos confirman el éxito que pueden tener las intervenciones breves para reducir las tendencias perfeccionistas (e.g., Bento, Pereira, Roque, Tavares, \& Ferreira, 2017). Esta conclusión es especialmente relevante por cuanto es sabido que el perfeccionismo es un rasgo de personalidad que tiende a ser relativamente estable a lo largo del tiempo (McGrath et al., 2012), resistente al tratamiento (Flett \& Hewitt, 2008) y que interfiere con el abordaje clínico de otros trastornos al perjudicar la adherencia a la terapia (Flett \& Hewitt, 2007). Parece ser entonces que, especialmente durante la infancia y la adolescencia, el perfeccionismo no es estático, sino que es sensible a estrategias de intervención que, aunque breves, pueden hacerle frente, prevenir sus efectos nocivos y, en general, inducir cambios positivos y duraderos sobre la personalidad. En consecuencia, es pertinente acompañar la sugerencia de diversos autores (e.g., Oros, 2005) con respecto a que la modificación de los elevados niveles de perfeccionismo mediante diversos programas debe tener un comienzo temprano.

De acuerdo con la teoría, la efectividad de la utilización terapéutica de los libros radica en los actos de lectura significativos que se producen a consecuencia de la asociación que se establece entre el mundo interior del lector y el texto (Arias et al., 2000). Más concretamente, debido a los lazos empáticos que el lector genera con los personajes de ficción, que sería lo que en última instancia le proporciona a aquel de un modo no invasivo- un modelo para afrontar las situaciones de conflicto (Stamps, 2003). Indudablemente, la experiencia que tuvo lugar brindó a los involucrados un enfoque menos amenazante para explorar los problemas asociados con la autocrítica, las presiones externas y el perfeccionismo poco saludable. Muestra del fenómeno de identificación con los personajes es que los participantes comenzaran a usar las palabras de aquellos durante los encuentros -e.g., "vamos pues...", "así es la vida", “... terrible, horrible, espantoso, horroroso"- y el momento catártico que tuvieron los niños al compartir las actitudes excesivamente críticas por parte de sus padres. Un factor que pudo haber alentado este proceso es el hecho de que la mayoría de las historias 
leídas -excepto Las jirafas no pueden bailar y Sofía, la vaca que amaba la músicainvolucraban como personaje principal a un niño -e.g., Petit, Beatriz, Vashti, Ramón, Alexander- con dificultades similares a las confrontadas por los participantes.

Por otra parte, durante el taller dictado se dedicó un tiempo considerable a la fase de discusión de las sesiones, y esta se complementó con actividades diseñadas para estimular el pensamiento creativo y la capacidad de resolver problemas. En este sentido, tanto las preguntas formuladas en las discusiones como las actividades posteriores buscaban que los participantes notaran que no estaban solos -que los demás también experimentaban sensaciones muy parecidas con relación a los temas tratados: el miedo al fracaso, el orden extremo, las expectativas infladas, etc.- y aprendieran que existían distintas soluciones a probar para los problemas que reportaban.

Como se había adelantado, es muy posible que los niños perfeccionistas tengan una mejor respuesta a la biblioterapia debido a que estos suelen ser estudiantes académicamente aventajados, por lo que quizás posean habilidades de comprensión lectora más avanzadas que las de sus compañeros, tales como hacer preguntas al texto, integrar la información y las experiencias que los libros describen con lecturas previas y con nuevos aprendizajes, intentar entender materiales de lectura desafiantes sin ser disuadidos por su dificultad, etcétera. A ello debe sumarse el entusiasmo por la lectura que este tipo de niños puede mostrar, condición bajo la cual se esperaría que los efectos de la biblioterapia se reforzaran aún más. De hecho, una de las razones por las que el uso de la biblioterapia no arrojó resultados significativos en algunos estudios (e.g., Weber, 1998) se debe a que no se aseguró el disfrute con los libros elegidos, un principio fundamental en su aplicación. En el estudio que el presente informe reporta, se cree que a los participantes les gustó selección de literatura empleada, además de que eran niños que en general solían disfrutar de la lectura, lo que contribuyó al alcance del objetivo que perseguía el taller de biblioterapia.

El presente estudio tiene varias limitaciones. En primer lugar debe considerarse la influencia que pudo tener la aplicación reiterada del instrumento de perfeccionismo, pues se ha conseguido que el solo hecho de repetir pruebas se puede asociar con mejoras significativas en medidas de personalidad (Hernández et al., 2014). En este mismo sentido, particular atención debe recibir el fenómeno conocido como regresión estadística, es decir, la tendencia de las puntuaciones extremas a revertirse -o regresarse- hacia la media de la distribución cuando se vuelve a medir (Hernández et al., 2014). Futuros estudios podrían incluir un grupo control para descartar los inconvenientes señalados como amenazas a la validez interna de la investigación. Lo que es más, se podría aumentar el control experimental trabajando con un diseño factorial donde la biblioterapia sea una de las opciones de tratamiento -variables independientes- para el perfeccionismo en niños.

Por otra parte, el número relativamente pequeño de participantes restringe la generalidad de los hallazgos. La cantidad de sujetos pudo haber sido un poco mayor, pero algunos abandonaron el estudio antes de su finalización, manifestando 
que si continuaban acudiendo perderían clases y se atrasarían en los contenidos académicos dictados. Según se supo, padres y docentes influyeron en dicha decisión, pues desalentaban la participación en el taller para priorizar la asistencia a clases. Esta circunstancia pone de relieve un problema de más amplio calado y es el hecho de que el foco de atención de los centros educativos lo ocupan usualmente las habilidades cognitivas, pese a haberse señalado que atender el desarrollo socioafectivo de los estudiantes favorece el éxito académico (Mena, Romagnoli, \& Valdés, 2009).

Por lo anterior, resulta de vital importancia brindar información a los padres y docentes sobre el perfeccionismo y sus posibles efectos negativos. En el caso de los educadores, una aspiración de mayor alcance sería capacitarlos en el uso de la biblioterapia en el aula, ya que es una técnica cuya utilidad en el tratamiento de problemas psicológicos está bien establecida, además de que puede jugar un papel importante en el desarrollo de habilidades comunicativas y en la elevación de la cultura general (Pardeck, 1995). La tarea requerida es aún más significativa con respecto a los padres, ya que las investigaciones acerca de las causas o los antecedentes del perfeccionismo muestran que las actitudes parentales pueden influir en los niveles de perfeccionismo de los hijos (e.g., Cook, 2012). En tal sentido, se recomienda que los padres participen en futuros programas centrados en el perfeccionismo.

Para el estudio realizado es difícil determinar qué partes del taller fueron más influyentes en los resultados. Se necesita mayor investigación para especificar qué componentes de la intervención son más valiosos y necesarios para producir resultados exitosos. Parece importante indicar que la propuesta aquí esbozada solo constituye una aproximación al uso terapéutico de los libros. Se esperaría que la misma fuese modificada y/o ampliada en cuanto a número de sesiones, cuentos empleados, videos presentados, pistas musicales escogidas y demás características de la organización experimental susceptibles de someterse a un proceso de validación por jueces expertos. De hecho, por razones de disponibilidad, otros libros aparentemente idóneos según el objetivo perseguido no se pudieron incluir en el taller. Se tiene confianza en que la efectividad de la biblioterapia puede extenderse más allá del conjunto de libros aquí presentados, pero sería deseable poder probarlo próximamente desde el punto de vista empírico. Por otro lado, hubo aspectos del perfeccionismo que, aunque planteados por los libros leídos, no pudieron ser evaluados con el instrumento del que se disponía. Es el caso de las dimensiones sociales del perfeccionismo -e.g., Presión Externa-, la obsesión por el orden -e.g., Compulsividad- y el perfeccionismo moral.

Finalmente, hay que tomar en cuenta que en los resultados de un experimento puede influir el punto en el tiempo en el que se completa la evaluación (Kazdin, 2001). En el estudio realizado, el postest se tomó inmediatamente después de la sesión de cierre del taller. Es posible que las conclusiones alcanzadas en este punto en el tiempo no se extiendan a un período posterior de días, semanas o meses. En definitiva, de lo que se trataría es de determinar si el perfeccionismo de los niños disminuye en sus escenarios cotidianos, donde están nuevamente a merced de las contingencias del entorno 
familiar; para muchos, las responsables del perfeccionismo infantil. Así que próximas investigaciones deberían incluir una medida de seguimiento para evaluar la estabilidad del cambio terapéutico.

Tanto la recomendación de contar con una fase de seguimiento, como la de que padres y maestros sean mediadores en el abordaje terapéutico del perfeccionismo en los niños, son sugerencias que tienen la mirada puesta en una orientación más ecológica en el desarrollo de mediciones e intervenciones (Campbell et al., 2000). Efectivamente, varios autores (Bieling, Israeli, Smith, \& Antony, 2003; Flett \& Hewitt, 2014; Vicent, Inglés et al., 2019a) han señalado la necesidad de que los modelos de tratamiento para el perfeccionismo infantil se prueben fuera de los ambientes clínicos, en situaciones reales de la vida diaria como las que proporciona el contexto escolar, uno de los contextos próximos en que el niño se desenvuelve y donde son especialmente relevantes las conductas perfeccionistas. La estrategia clínico-educativa aquí implementada no permaneció ajena a este desafío y representa un intento por allanar el camino en esa dirección.

Aun con sus limitaciones, este trabajo tiene el mérito de ser - hasta donde se sabeel primero en realizarse en Latinoamérica y con participantes de habla hispana, para corroborar la utilidad de la biblioterapia como tratamiento destinado a los niños perfeccionistas. Se recomienda la aplicación de la denominada terapia de lectura en contextos semejantes al aquí descrito.

\section{Referencias}

Aguilar, L. (2019). Perfeccionismo en escolares de Caracas: diferencias en función del sexo, tipo de institución educativa y nivel de estrés. Revista de Psicología de la Universidad de Antioquia, 11(1), 7-33. doi: 10.17533/udea.rp.v11n1a01

Aguilar, L., \& Castellanos, M. (2016a). Adaptación lingüístico-cultural de una escala argentina de perfeccionismo infantil al contexto venezolano. Revista Electrónica de Metodología Aplicada, 21(2), 1-32. doi: 10.17811/rema.21.2.2016.1-32

Aguilar, L., \& Castellanos, M. (2016b). Validación de contenido de una versión culturalmente adaptada al contexto venezolano de la Escala de Perfeccionismo Infantil de Oros.
Pensando Psicología, 12(20), 2941. doi: $10.16925 /$ pe.v12i20.1561

Aguilar, L., \& Castellanos, M. (2017). Validación psicométrica de una escala de perfeccionismo infantil en niños venezolanos. Psicoespacios, 11(18), 9-42. doi: $10.25057 / 21452776.875$

Andreae, G., \& Parker-Rees, G. (1999/2009). Las jirafas no pueden bailar. Madrid: Bruño.

Arias, M., Miguez, L., Molfino, E., \& Rega, L. (2000). Biblioterapia. Información, cultura y sociedad, 2, 85-93. doi: 10.34096\%2Fics. i2. 1036

Babarro, I., \& Lacalle, J. (2018). La literatura como instrumento terapéutico en el proceso salud-enfermedad durante 
la infancia. Enfermería Global, 50, 585-600. doi: 10.6018/ eglobal.17.2.299201

Bento, C., Pereira, A., Roque, C., Tavares, J., \& Ferreira, A. (2017). Longitudinal effects of an intervention on perfectionism in adolescents. Psicothema, 29(3), 317-322. doi: 10.7334/ psicothema2016.223

Bieling, P., Israeli, A., Smith, J., \& Antony, M. (2003). Making the grade: The behavioural consequences of perfectionism in the classroom. Personality and Individual Differences, 35(1), 163-178. doi: 10.1016/s0191-8869(02)00173-3

Bong, M., Hwang, A., Noh, A., \& Kim, S. (2014). Perfectionism and motivation of adolescents in academic contexts. Journal of Educational Psychology, 106(3), 711-729. doi: $10.1037 / \mathrm{a} 0035836$

Botelho, R. (2013). Las funciones de la literatura infantil en la Educación. Revista Iberoamericana de Educación, 61(3). doi: 10.35362/ rie6131080

Bueno, S., \& Caldin, C. (2002). A aplicação da biblioterapia em crianças. Revista ACB, 7(2), 157-170. Recuperado de http:// revista.acbsc.org.br/racb/article/ view/372

Camero, N. (2016). Estado del arte de las experiencias aplicadas de biblioterapia en América Latina (Trabajo de grado, Universidad de La Salle, Bogotá, Colombia). Recuperado de http://repository.lasalle.edu.co/ handle/10185/20833

Campbell, M., Fitzpatrick, R., Haines, A., Kinmonth, A., Sandercock, P., Spiegelhalter, D., Tyrer, P. (2000). Framework for design and evaluation of complex interventions to improve health. BMJ, 321, 694-696. doi: 10.1136/ bmj.321.7262.694

Castro, A., \& Altamirano, N. (2016). ¿Leer para estar bien?: prácticas actuales y perspectivas sobre la biblioterapia como estrategia educativo-terapéutica. Investigación bibliotecológica, 32(74), 171-192. doi: 10.22201/ iibi.24488321xe. 2018.74 .57918

Chemisquy, S. (2018). Autopresentación perfeccionista, perfeccionismo $y$ sentimientos depresivos en niños: análisis del efecto mediador de la soledad y el apoyo social percibido. Manuscrito inédito, Universidad de la Cuenca del Plata, Corrientes, Argentina.

Chemisquy, S., Oros, L., Serppe, M., \& Ernst, C. (2019). Caracterización del perfeccionismo disfuncional en la niñez tardía. Apuntes Universitarios, 9(2), 1-26. doi: 10.17162/au.v9i2.355

Cook, L. (2012). The influence of parent factors on child perfectionism: A cross-sectional study (Tesis doctoral, University of Nevada, Las Vegas). Recuperada de http://digitalscholarship. unlv.edu/cgi/viewcontent. c gi ? article $=2552 \& \mathrm{con}$ ntext $=$ thesesdissertations

De Pennart, G. (1999/2011). Sofia, la vaca 
que amaba la música. Barcelona: Corimbo.

Douilliez, C., \& Hénot, E. (2013). Mesures du perfectionnisme chez l'adolescent: Validation des versions Francophones de deux questionnaires. Canadian Journal of Behavioural Science, 45(1), 64-71. doi: 10.1037/a0022686

Dry, S., Kane, R., \& Rooney, R. (2015). An investigation into the role of coping in preventing depression associated with perfectionism in preadolescent children. Frontiers in Public Health, 3. doi: 10.3389/ fpubh.2015.00190

Dunlap, W., Cortina, J., Vaslow, J., \& Burke, M. (1996). Meta-analysis of experiments with matched groups or repeated measures designs. Psychological Methods, 1(2), 170-177. doi: 10.1037/1082989X.1.2.170

Egan, S., Piek, J., Dyck, M., \& Rees, C. (2007). The role of dichotomous thinking and rigidity in perfectionism. Behaviour Research and Therapy, 45(8), 1813-1822. doi: 10.1016/j. brat.2007.02.002

Elizathe, L., Murawski, B., Custodio, J., \& Rutsztein, G. (2012). Riesgo de trastorno alimentario en niños escolarizados de Buenos Aires: su asociación con perfeccionismo. Revista Mexicana de Trastornos Alimentarios, 3, 106-120. Recuperado de http://journals. iztacala.unam.mx/index.php/ amta/article/view/226

Elizathe, L., Murawski, B., Lievendag,
L., Diez, M., Barrios, R., Cruz, G., \& Rutsztein, G. (2011). Riesgo de trastorno alimentario y perfeccionismo en niños y niñas escolarizados. En N. Cervone (Presidencia), Memorias del III Congreso Internacional de Investigación y Práctica Profesional en Psicología - XVIII Jornadas de Investigación - VII Encuentro de Investigadores en Psicología del MERCOSUR (p. 293). Buenos Aires, Argentina: UBA.

Flett, G., \& Hewitt, P. (2002). Perfectionism: Theory, research and treatment. Washington, DC: American Psychological Association.

Flett, G., \& Hewitt, P. (2007). Cognitive and self-regulation aspects of perfectionism and their implications for treatment: Introduction to the special issue. Journal of Rational-Emotive \& Cognitive-Behavior Therapy, 25(4), 227-236. doi: 10.1007/ s10942-007-0054-5

Flett, G., \& Hewitt, P. (2008). Treatment interventions for perfectionism-A cognitive perspective: Introduction to the special issue. Journal of RationalEmotive \& Cognitive-Behavior Therapy, 26(3), 127-133. doi: 10.1007/s10942-007-0063-4

Flett, G., \& Hewitt, P. (2012). Perfectionism and cognitive factors in distress and dysfunction in children and adolescents: Introduction to the special issue. Journal of Rational- 
Emotive \& Cognitive-Behavior Therapy, 30(2), 53-61. doi: 10.1007/s10942-011-0134-4

Flett, G., \& Hewitt, P. (2013). Disguised distress in children and adolescents "flying under the radar": Why psychological problems are underestimated and how schools must respond. Journal of Rational-Emotive and CognitiveBehavior Therapy, 28(1), 12-27. doi: $10.1177 / 0829573512468845$

Flett, G., \& Hewitt, P. (2014). A proposed framework for preventing perfectionism and promoting resilience and mental health among vulnerable children and adolescents. Psychology in the Schools, 51(9), 899-912. doi: 10.1002/pits.21792

García-Fernández, J., Vicent, M., Inglés, C., Gonzálvez, C., \& Sanmartín, R. (2017). Relación entre el perfeccionismo socialmente prescrito y la conducta agresiva durante la infancia tardía. European Journal of Education and Psychology, 10(1), 15-22. doi: 10.1016/j.ejeps.2016.10.003

Gómez, K. (2011). La biblioterapia como práctica profesional del bibliotecólogo (Trabajo de grado de licenciatura, Universidad Central de Venezuela, Caracas). Recuperado de http://eprints.rclis. org $/ 15583 /$

Gonzálvez, C., Inglés, C., Lagos-San Martín, N., García-Fernández, J., \& Martínez-Monteagudo, M. (2015). Análisis bibliométrico sobre el perfeccionismo en la infancia y la adolescencia.

International Journal of Developmental and Educational Psychology, 1(1), 211-218. doi: 10.17060/ijodaep.2015.n1.v1.48

Gonzálvez, C., Sanmartín, R., GarcíaFernández, J., Inglés, C., \& Vicent, M. (2016). Perfeccionismo socialmente prescrito como predictor del alto rechazo a la escuela. International Journal of Developmental and Educational Psychology, 1(1), 25-32. doi: 10.17060/ijodaep.2016.n1.v1.185 Hernández, R., Fernández, C., \& Baptista, P. (2014). Metodología de la investigación ( $6^{\mathrm{a}}$ ed.). México: McGraw-Hill Interamericana.

Hewitt, P., Caelian, C., Flett, G., Sherry, S., Collins, L., \& Flynn, C. (2002). Perfectionism in children: Associations with depression, anxiety, and anger. Personality and Individual Differences, 32(6), 1049-1061. doi: 10.1016/s01918869(01)00109-x

Hewitt, P., Flett, G., \& Mikail, S. (2017). Perfectionism: A relational approach to conceptualization, assessment, and treatment. Nueva York: The Guildford Press.

Huggins, L., Davis, M., Rooney, R., \& Kane, R. (2008). Socially prescribed and self-oriented perfectionism as predictors of depressive diagnosis in preadolescents. Australian Journal of Guidance and Counselling, 18(2), 182-194. doi: 10.1375/ajgc.18.2.182

İlter, B., Leana-Taşcılar, M., \& Saltukoğlu, G. (2018). The effects of the 
bibliotherapy technique on perfectionism levels in gifted children: An experimental study. European Journal of Education Studies, 4(12), 153-176. doi: 10.5281/zenodo. 1343945

Inglés, C., García-Fernández, J., Vicent, M., Gonzálvez, C., \& Sanmartín, R. (2016). Profiles of perfectionism and school anxiety: A review of the $2 \times 2$ model of dispositional perfectionism in child population. Frontiers in Psychology, 7. doi: 10.3389/fpsyg.2016.01403

Kazdin, A. (2001). Métodos de investigación en psicología clínica ( $3^{\mathrm{a}}$ ed.). México: Pearson Educación.

Macbeth, G., Razumiejczyk, E., \& Ledesma, R. (2011). Cliff's Delta Calculator: A non-parametric effect size program for two groups of observations. Universitas Psychologica, 10(2), 545-555. doi: $10.11144 / J a v e r i a n a . u p s y 10-$ 2.cdcp

Marín,A. (2009). Diseño de un programa de biblioterapia para niños víctimas de maltrato (Trabajo de grado de licenciatura inédito). Universidad Central de Venezuela, Caracas.

McGrath, D., Sherry, S., Stewart, S., Mushquash, A., Allen, S., Nealis, L., \& Sherry, D. (2012). Reciprocal relations between self-critical perfectionism and depressive symptoms: Evidence from a short-term, four-wave longitudinal study. Canadian Journal of Behavioural Science, 44(3), 169-181. doi: 10.1037/ a0027764

Mena, M., Romagnoli, C., \& Valdés, A. (2009). El impacto del desarrollo de habilidades socio afectivas y éticas en la escuela. Actualidades Investigativas en Educación, 9(3). doi: 10.15517/AIE.V9I3.9507

Mitchell, J., Newall, C., Broeren, S., \& Hudson, J. (2013). The role of perfectionism in cognitive behaviour therapy outcomes for clinically anxious children. Behaviour Research and Therapy, 51(9), 547-554. doi: 10.1016/j. brat.2013.05.015

Misenta, M. (2010). Petit, el monstruo. Santiago: Ocho Libros Editores.

Mofield, E., \& Chakraborti-Ghosh, S. (2010). Addressing multidimensional perfectionism in gifted adolescents with affective curriculum. Journal for the Education of the Gifted, 33(4), 479-513. doi: 10.1177/016235321003300403

Montero, I., \& León, O. (2007). A guide for naming research studies in Psychology. International Journal of Clinical and Health Psychology, 7(3), 847-862. Recuperado de http://www.aepc. es/ijchp/articulos_pdf/ijchp-256. pdf

Montgomery, W. (2002). Ingeniería del comportamiento. Aplicaciones clínicas y educativas. Lima: Asociación Peruana de Psicología Interconductual (ASSPSI).

Morán, C., García, C., \& Fínez, M. (2015). Acoso escolar: relación con sentimiento de soledad 
y perfeccionismo positivo en adolescentes. International Journal of Developmental and Educational Psychology, 1(1), 199-210. doi: 10.17060/ijodaep.2015.n1.v1.62

Morris, L., \& Lomax, C. (2014). Assessment, development, and treatment of childhood perfectionism: A systematic review. Child and Adolescent Mental Health, 19(4), 225-234. doi: 10.1111/camh.12067

Nobel, R. (2007). Perfectionism in schoolage children experiencing symptoms of depression and anxiety: Characterization and relationship to treatment outcomes (Tesis doctoral inédita). University of Toronto, Canadá.

Nobel, R., Manassis, K., \& WilanskyTraynor, P. (2012). The role of perfectionism in relation to an intervention to reduce anxious and depressive symptoms in children. Journal of Rational-Emotive \& Cognitive-Behavior Therapy, 30(2), 77-90. doi: 10.1007/s10942011-0133-5

Nugent, S. (2000). Perfectionism: Its manifestations and classroombased interventions. Journal of Secondary Gifted Education, 11(4), 215-221. doi: 10.4219/jsge-2000630

Oros, L. (2003). Medición del perfeccionismo infantil: desarrollo y validación de una escala para niños de 8 a 13 años de edad. Revista Iberoamericana de Diagnóstico y Evaluación Psicológica, 16(2), 99-112. Recuperado de http://www.aidep. org/03_ridep/R16/R166.pdf
Oros, L. (2005). Implicaciones del perfeccionismo infantil sobre el bienestar psicológico: orientaciones para el diagnóstico y la práctica clínica. Anales de Psicología, 21(2), 294-303. Recuperado de http://revistas.um.es/analesps/ article/view/26951

Pardeck, J. (1995). Bibliotherapy: An innovative approach for helping children. Early Child Development and Care, 110(1), 83-88. doi: 10.1080/0300443951100106

Pérez de Rosell, M. (2011). Literapia como práctica terapéutica de los estudiantes de Autodesarrollo del DCS en el Hospital Universitario de Pediatría "Agustín Zubillaga" de Barquisimeto. Una experiencia educativa. Boletín Médico de Postgrado, 27(3), 109-117. Recuperado de http://bibvirtual. u cla.edu.ve/c g i - w in / b e alex.?Acceso $=$ T070200000150/9 \& Nombrebd=BOLMED\&TipoDoc $=$ S\&Sesion $=2003959721$

Pett, M., \& Rubinstein, G. (2013). La niña que nunca cometía errores. Barcelona: Obelisco.

Prieto, G., \& Muñiz, J. (2000). Un modelo para evaluar la calidad de los tests utilizados en España. Papeles del Psicólogo, 77, 65-72. Recuperado de http://www.papelesdelpsicologo. es/resumen?pii $=1102$

Ramírez, A., \& Ramírez, C. (2005). Así es la vida. Valencia: Diálogo.

Reynolds, P. (2003). El punto. Barcelona: Serres (RBA Libros).

Reynolds, P. (2004). Casi. Barcelona: Serres (RBA Libros). 
Rincón, L. (2013). Estado del arte y la fundamentación teórica y práctica del uso de la biblioterapia en la recuperación y el bienestar de pacientes en los centros de salud (Trabajo de grado, Pontificia Universidad Javeriana, Bogotá, Colombia). Recuperado de http:// repository.javeriana.edu.co/ handle/10554/11269

Santalla, Z., \& González, M. (2011). Principios éticos de la investigación. En Z. Santalla (Ed.), Introducción a la metodología de investigación en psicología (pp. 357-374). Caracas: UCAB.

Shafran, R., Egan, S., \& Wade, T. (2010). Overcoming perfectionism: $A$ self-help guide using cognitivebehavioural techniques. Londres: Constable and Robinson.

Silverberg, L. (2003). Bibliotherapy: The therapeutic use of didactic and literary texts in treatment, diagnosis, prevention, and training. The Journal of the American Osteopathic Association, 103(3), 131-135.

Somov, P. (2010). Present perfect: A mindfulness approach to letting go of perfectionism and the need for control. Oakland: New Harbinger Publications.

Soreni, N., Streiner, D., McCabe, R., Bullard, C., Swinson, R., Greco, A.,... Szatmari, P. (2014). Dimensions of perfectionism in children and adolescents with obsessivecompulsive disorder. Journal of the Canadian Academy of Child and Adolescent Psychiatry, 23(2), 136-
143.

Stamps, L. (2003). Bibliotherapy: How books can help students cope with concerns and conflicts. The Delta Kappa Gamma Bulletin, 70(1), 2529.

Stoeber, J., Schneider, N., Hussain, R., \& Matthews, K. (2014). Perfectionism and negative affect after repeated failure: Anxiety, depression, and anger. Journal of Individual Differences, 35(2), 87-94. doi: 10.1027/1614-0001/a000130

Stornelli, D., Flett, G., \& Hewitt, P. (2009). Perfectionism, achievement, and affect in children: A comparison of students from gifted, arts, and regular programs. Canadian Journal of School Psychology, 24(4), 267-283. doi: 10.1177/0829573509342392

Vicent, M. (2017). Estudio del perfeccionismo y su relación con variables psicoeducativas en la infancia tardia (Tesis doctoral, Universitat d'Alacant, España). Recuperada de http://rua.ua.es/ dspace/handle/10045/73818

Vicent, M., Gonzálvez, C., Sanmartín, R., Fernández-Sogorb, A., CarguaGarcía, N., \& García-Fernández, J. (2019). Perfectionism and school anxiety: More evidence about the $2 \times 2$ model of perfectionism in an Ecuadorian population. School Psychology International, 40(5), 474-492. doi: $10.1177 / 0143034319859047$

Vicent, M., Inglés, C., Gonzálvez, C., Sanmartín, R., Aparicio-Flores, M., \& García-Fernández, J. (2019a). Perfiles de perfeccionismo 
y autoatribuciones causales académicas en estudiantes españoles de Educación Primaria. Revista de Psicodidáctica, 24(2), 103-110. doi: 10.1016/j.psicod.2019.01.001

Vicent, M., Inglés, C., Gonzálvez, C., Sanmartín, R., Aparicio-Flores, M., \& García-Fernández, J. (2019b). Self-criticism, strivings and aggressive behavior in Spanish children: The two sides of self-oriented perfectionism. The Spanish Journal of Psychology, 22. doi: 10.1017/sjp.2019.29

Vicent, M., Inglés, C., Sanmartín, R., Gonzálvez, C., \& García-Fernández, J. (2018). Aggression profiles in the Spanish child population: Differences in perfectionism, school refusal and affect. Frontiers in Behavioral Neuroscience, 12. doi: 10.3389/fnbeh.2018.00012

Viorst, J., \& Cruz, R. (1972/1991). Alexander y el día terrible, horrible, espantoso, horroroso. Nueva York: Live Oak Media.

Weber, D. (1998). The influence of bibliotherapy on genderrole attitudes of gifted female adolescents enrolled in a magnet program for the gifted (Tesis doctoral inédita). University of Alabama, EE. UU.

Wimberley, T., Mintz, L., \& Suh H. (2016). Perfectionism and mindfulness: Effectiveness of a bibliotherapy intervention. Mindfulness, 7(2), 433-444. doi: 10.1007/s12671-0150460-1

Wolters, L., Hogendoorn, S., Koolstra, T., Vervoort, L., Boer, F., Prins, P., \& de Haan, E. (2011). Psychometric properties of a Dutch version of the Obsessive Beliefs QuestionnaireChild Version (OBQ-CV). Journal of Anxiety Disorders, 25(5), 714-721. doi: 10.1016/j. janxdis.2011.03.008

Zousel, M., Rule, A., \& Logan, S. (2013). Teaching primary grade students perfectionism through cartoons compared to bibliotherapy. International Electronic Journal of Elementary Education, 5(2), 199218. Recuperado de http://www. iejee.com/index.php/IEJEE/article/ view/21 